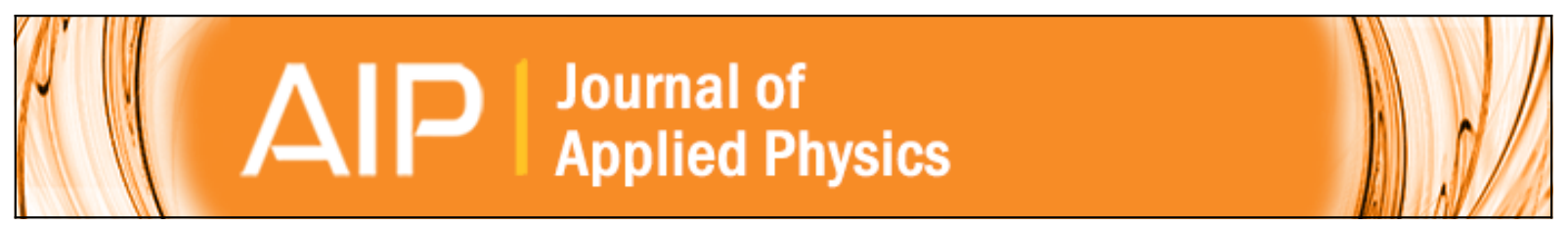

\title{
Flux distraction effect on magnetoelectric laminate sensors and gradiometer
}

Ying Shen, Junqi Gao, Yaojin Wang, Davresh Hasanyan, Peter Finkel, Jiefang Li, and D. Viehland

Citation: Journal of Applied Physics 114, 134104 (2013); doi: 10.1063/1.4824184

View online: http://dx.doi.org/10.1063/1.4824184

View Table of Contents: http://scitation.aip.org/content/aip/journal/jap/114/13?ver=pdfcov

Published by the AIP Publishing

\section{Articles you may be interested in}

Dependence of magnetic field sensitivity of a magnetoelectric laminate sensor pair on separation distance: Effect of mutual inductance

J. Appl. Phys. 111, 033923 (2012); 10.1063/1.3684986

Magnetoelectric nonlinearity in magnetoelectric laminate sensors

J. Appl. Phys. 110, 114510 (2011); 10.1063/1.3665130

Metal-ceramic laminate composite magnetoelectric gradiometer

Rev. Sci. Instrum. 81, 033906 (2010); 10.1063/1.3354985

Geomagnetic sensor based on giant magnetoelectric effect

Appl. Phys. Lett. 91, 123513 (2007); 10.1063/1.2789391

Vortex magnetic field sensor based on ring-type magnetoelectric laminate

Appl. Phys. Lett. 85, 2307 (2004); 10.1063/1.1791732

MIT LINCOLN

LABORATORY CAREERS

Discover the satisfaction of innovation and service

to the nation
- Space Control

- Air \& Missile Defense

- Communications Systems \& Cyber Security

- Intelligence, Surveillance and

Reconnaissance Systems

- Advanced
Electronics
- Tactical Systems
- Homeland
Protection
- Air Traffic Control

LINCOLN LABORATORY

MassachusetTs Institute of TeChNOLOgY

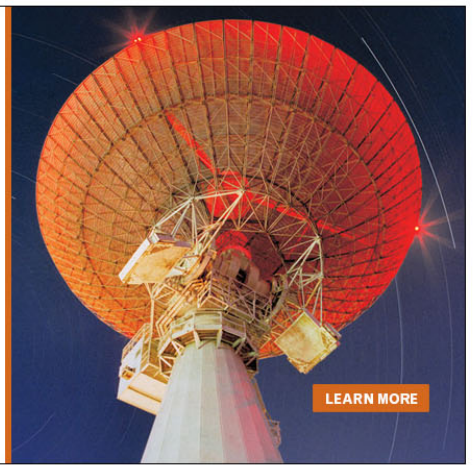




\title{
Flux distraction effect on magnetoelectric laminate sensors and gradiometer
}

\author{
Ying Shen, ${ }^{1}$ Junqi Gao, ${ }^{1}$ Yaojin Wang, ${ }^{1}$ Davresh Hasanyan, ${ }^{1}$ Peter Finkel, ${ }^{2}$ Jiefang Li, ${ }^{1}$ \\ and D. Viehland ${ }^{1}$ \\ ${ }^{1}$ Department of Materials Science and Engineering, Virginia Tech, Blacksburg, Virginia 24061, USA \\ ${ }^{2}$ Naval Undersea Warfare Center, Newport, Rhode Island 02840, USA
}

(Received 7 September 2013; accepted 17 September 2013; published online 4 October 2013)

\begin{abstract}
A magnetic flux distraction effect caused by a nearby metallic material was investigated for Metglas/ $\mathrm{Pb}\left(\mathrm{Mg}_{1 / 3} \mathrm{Nb}_{2 / 3}\right) \mathrm{O}_{3}-\mathrm{PbTiO}_{3}$ laminated magnetoelectric (ME) sensors. Using flux distraction, a ME sensor can perform an accurate search for metallic targets of different dimensions at various distances. Detection results and simulations were in good agreement. The findings demonstrate an effective means to employ stationary ME sensors and gradiometers for magnetic search applications. (C) 2013 AIP Publishing LLC. [http://dx.doi.org/10.1063/1.4824184]
\end{abstract}

The magnetoelectric (ME) effect is the induction of polarization by an applied magnetic field or vice versa. ${ }^{1} \mathrm{ME}$ laminated heterostructures have generally been comprised of two phases: magnetostrictive and piezoelectric ones, where the two different ferroic degrees of freedom interact with each other by elastic forces resulting in a product ME tensor effect. ${ }^{2}$ For ME sensors, Metglas is a desirable candidate for the magnetostrictive phase, due to its large effective piezomagnetic coefficient and low required DC magnetic bias. Metglas/ $\mathrm{Pb}\left(\mathrm{Mg}_{1 / 3} \mathrm{Nb}_{2 / 3}\right) \mathrm{O}_{3}-\mathrm{PbTiO}_{3}$ ME laminate sensors with pico-Tesla (pT) noise floors at $1 \mathrm{~Hz}$ have been reported, demonstrating an alternative magnetometer technology that is passive and that operates at room-temperature. ${ }^{3}$ However, in case of moving ME sensors, vibration noise might be introduced. This might undermine the high sensitivity of its normal stationary state and could be an important issue with regards to applications. ${ }^{4}$

Due to a high relative permeability of $\mu_{\mathrm{r}}>40000$, Metglas results in a significant magnetic flux concentration effect into the ME laminates. ${ }^{5}$ Indeed, by optimizing the geometry and volume ratio of Metglas, an increased flux concentration effect has been exploited for enhancing the effective ME coefficient and sensitivity of ME laminates. ${ }^{5,6}$ Alternatively, a flux distraction effect in Metglas-based ME sensors could be used to effectively address limitations in detecting unmoved magnetic targets over a specific survey area, while at the same time allowing the ME sensor to remain stationary. Magnetic flux concentration effects in Metglas can be notably changed by adjacent high-mu objects, in particular when they are located in the vicinity of a magnetic field. A certain amount of the flux that was originally captured by the Metglas would then be shunted to the other high permeability material, producing the flux distraction, and in-turn, an apparent reduced ME response. This finding properly presents a means to enable ME sensors in different applications, such as non-destructive testing of constructions, intelligent transportation systems, ${ }^{7}$ archeological prospecting, and underground natural resources extraction. ${ }^{8}$ However, there has been little previous work in this field of study.

Here, such magnetic flux distraction effect has been investigated. Two essential parameters (the object volume, and the distance between sensor and object) that affect flux distraction have also been analyzed. To reject environmental noises, two identical sensors were used to form a gradiometer pair whose differential output was able to effectively mitigate the environmental noise. Two separately placed sensors with a particular baseline should have the same level of response to a far-field environmental noise, but they will have notably different magnetic field amplitudes, as the magnetic anomaly field drops in intensity as the cube of the distance. ${ }^{4}$ Thus, a ME gradiometer with a vertical gradient $\mathrm{dB}_{\mathrm{x}} / \mathrm{dx}$ was developed which was capable of a high detection sensitivity of $60 \mathrm{pT} / \sqrt{ } \mathrm{Hz}$ at quasi-static frequencies, especially in an open environment.

A magnetostatic simulation was performed using a finite element model via a Comsol 4.2 AC/DC Module for a coil, ME sensor, and a metal plate object. An AC magnetic field $H_{\mathrm{AC}}$ was simulated using a solenoid coil driven along the horizontal $x-y$ plane. Assuming a static current density denoted as $J$, the magnetic vector potential $A$ must satisfy the vector Poisson equation

$$
\nabla^{2} A=-\mu J
$$

where the relative permeability of air is $\mu_{r, a i r}=1$ and that of the Metglas-based sensor is $\mu_{r \text {,sensor }}=40000$. Equation (1) can be decomposed into three scalar Poisson equations

$$
\left\{\begin{array}{l}
\nabla^{2} A_{x}=-\mu J_{x} \\
\nabla^{2} A_{y}=-\mu J_{y} \\
\nabla^{2} A_{z}=-\mu J_{z} .
\end{array}\right.
$$

The relation between fields and potentials is then given by

$$
B=\nabla \times A .
$$

Figure 1(a) shows a simulation schematic diagram for the magnetic flux density of the ME sensor to a magnetic induction field, via 147000 mesh points within a controlled sphere volume. One can see that the flux is relatively intense close to the edges of the coil and decays rapidly with increasing distance from the coil. It can be also seen that the flux concentration effect is quite significant in the ME sensor due to the high permeability of the Metglas layers. The simulated 

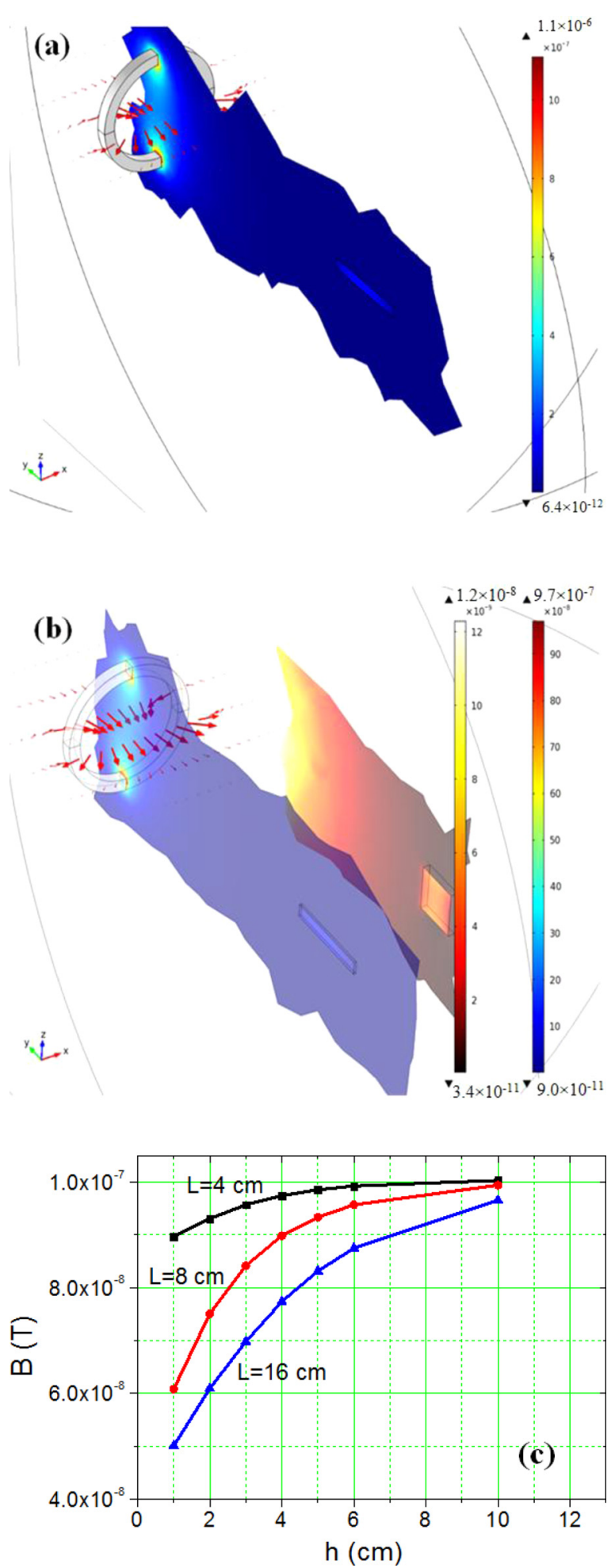

FIG. 1. (a) Finite element modeling simulation schematic diagram for magnetic flux density of the ME sensor to an induction magnetic field in the absence of a metal object. (b) Simulation diagram for magnetic flux distraction effect caused by the introduction of an adjacent high-mu metal object. (c) Estimated magnetic field response of a ME sensor as a function of distance $\mathrm{h}$ for different length $\mathrm{L}$ of close metal plates.

averaged magnetic flux density of the ME sensor was 100.34 nano-Tesla. Figure 1(b) illustrates the magnetic flux distraction effect caused by the introduction of an adjacent high-mu metal with permeability $\mu_{r, \text { metal }}=200$. One can see that the metal extracts flux density due to its relatively high permeability, leaving the sensor capturing less flux than before (i.e., compare to Fig. 1(a)). Figure 1(c) shows a summary of the volume averaged magnetic flux density of the ME sensor in the presence of a thin metal plate as a function of distance (h) between them for metal plates of different lengths (L) (i.e., fixed width of $4 \mathrm{~cm}$ and thickness of $0.3 \mathrm{~cm}$ ). From this figure, it can be seen for $\mathrm{L}=4 \mathrm{~cm}$ that the magnetic field response was nearly unchanged at a value of around $100 \mathrm{nT}$ for $\mathrm{h} \geq 10 \mathrm{~cm}$; initially slowly decreased as $\mathrm{h}$ decreased; and then decreased more rapidly as $\mathrm{h}$ was further decreased to $1 \mathrm{~cm}$. Clearly, the magnetic field response decreased with increasing length of the metal plate from $\mathrm{L}=4 \mathrm{~cm}, 8 \mathrm{~cm}$ to $16 \mathrm{~cm}$.

An experiment was then conducted with ME laminates composed of six layers of magnetostrictive Metglas laminated onto a core piezoelectric PMN-PT layer. An incident magnetic field induced the Metglas to produce a strain through magnetostriction, which in turn generated a mechanicalstrain-induced electric signal in the composite core via piezoelectricity. ${ }^{9}$ The core composites were two Kapton interdigitated copper electrode layers with $500 \mu \mathrm{m}$ wide digits spaced at $1.5 \mathrm{~mm}$ center-to-center (Smart materials, USA) fixed to both top and bottom surfaces of five $40 \times 2 \times 0.2 \mathrm{~mm}^{3}$ PMN-PT fibers (Ceracomp, Korean) using epoxy resin (Stycast 1264, USA) to obtain a push-pull geometry. The $25 \mu \mathrm{m}$ Metglas foils (Vacuumscheltze $\mathrm{GmbH} \& \mathrm{Co} . \mathrm{KG}$, Germany) with a saturation magnetostriction of about $27 \mathrm{ppm}$ were cut to a surface area of $80 \times 10 \mathrm{~mm}^{2}$ in order to match the total width of the five PMN-PT fibers. ${ }^{10}$ A length of $80 \mathrm{~mm}$ was chosen as a trade-off between maximum flux concentration and practical sensor size. ${ }^{11}$ Three layers of such Metglas pieces were stacked one on top of each other using the epoxy resin (West system 206, USA) and then two three-layer Metglas stacks were symmetrically attached to the top and bottom sides of the electrode-(PMN-PT)-electrode core using the same epoxy. The laminates were pressed by a vacuum bag method to minimize the epoxy thickness in-between the layers and cured for more than $24 \mathrm{~h}$ at room temperature to form the ME laminates.

The ME laminates and charge amplifiers (CAs) circuits were then assembled into a plastic housing enclosure to form ME sensor units. The CAs were designed with a uniform gain transfer function of around $1 \mathrm{~V} /$ pico-Coulomb (pC) over a frequency bandwidth of $0.6-10 \mathrm{~Hz}{ }^{12}$ Two permanent magnets were placed at either end of the ME laminates along the longitudinal axis in order to achieve the maximum ME coefficient as well as the highest sensitivity to minute magnetic field variations. Figure 2(a) shows the measured and estimated equivalent magnetic noise floor of the ME laminates for $1<f<100 \mathrm{~Hz}$ in a zero-Gauss, vibration isolation chamber. (The gain factor between $10-100 \mathrm{~Hz}$ has been normalized) A very low equivalent magnetic noise of $7.8 \mathrm{pT} /{ }_{\sqrt{ }} \mathrm{Hz}$ was found at $f=1 \mathrm{~Hz}$ that was close to the predicted value of $7.2 \mathrm{pT} / \sqrt{ } \mathrm{Hz}$. The prediction method was according to that precisely reported. ${ }^{13}$ It should be noted that the noise floor was about $1 \mathrm{pT} /{ }_{\sqrt{ }} \mathrm{Hz}$ at higher frequencies. The very low equivalent magnetic noise makes the ME laminate sensor promising for use in ultralow magnetic field applications. 

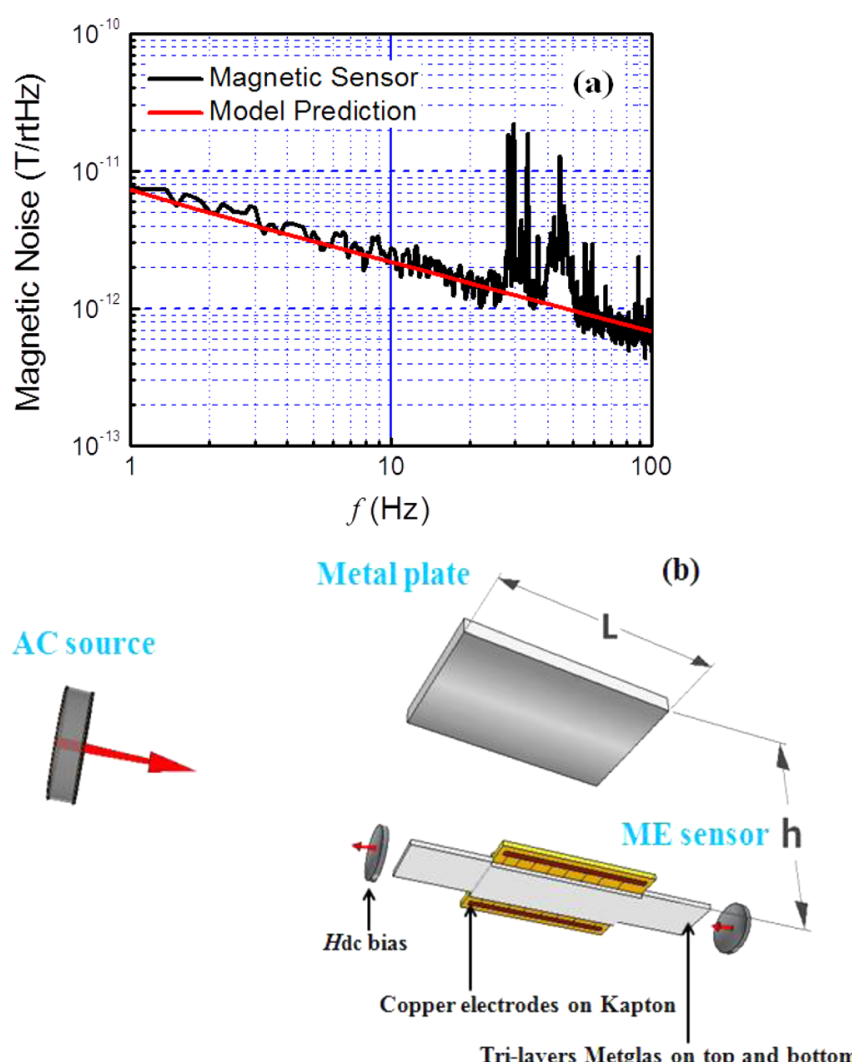

FIG. 2. (a) Measured and estimated equivalent magnetic noise floor of the ME laminates in the frequency range of $1<f<100 \mathrm{~Hz}$ taken in a zeroGauss, vibration isolation chamber. (b) Schematic representation of a signal generator (solenoid coil), ME sensor device, and a thin metal plate of length $\mathrm{L}$. The sensor and metal are separated by a distance $\mathrm{h}$.

Figure 2(b) illustrates the configuration of a signal generator (a solenoid coil), ME sensor unit, and a target metal plate of the same dimension and relative permeability as the module. The sensor and the metal plate were placed parallel to each other with their core points aligned along the transverse axes. The distance between the sensor and the metal plate was defined as $h$, and the length of the metal plate was represented by L. A coil located $40 \mathrm{~cm}$ away from the sensor was driven at a frequency of $7 \mathrm{~Hz}$ by a current density of $J=230 \mathrm{~A} / \mathrm{m}^{2}$, generating a constant sinusoid output. A data logger (Campbell Scientific, Inc., USA) with a $100 \mathrm{~Hz}$ sampling rate was employed to collect the signal from the CAs, and the output signal was then processed by Matlab. Experiments were performed in an open laboratory environment which can be considered as having high magnetic and vibration disturbances.

Figure 3(a) shows a sequence of magnetic field responses as a function of $h$ for different lengths $(L)$ of the metal plate. From this figure, it can be seen for $\mathrm{L}=4 \mathrm{~cm}$ that the magnetic field response was nearly unchanged at $101.4 \mathrm{nT}$ at distance of $\mathrm{h} \geq 10 \mathrm{~cm}$; initially decreased slowly as $\mathrm{h}$ was decreased; and then subsequently decreased more rapidly as $\mathrm{h}$ was further decreased to $1 \mathrm{~cm}$. It can also be seen that the magnetic field response decreased with increasing length of the metal plate for $4 \leq \mathrm{L} \leq 16 \mathrm{~cm}$ in a manner nearly equal to the simulated results (i.e., compare with Fig. 1(c)). Figure 3(b) shows the magnetic field decrement
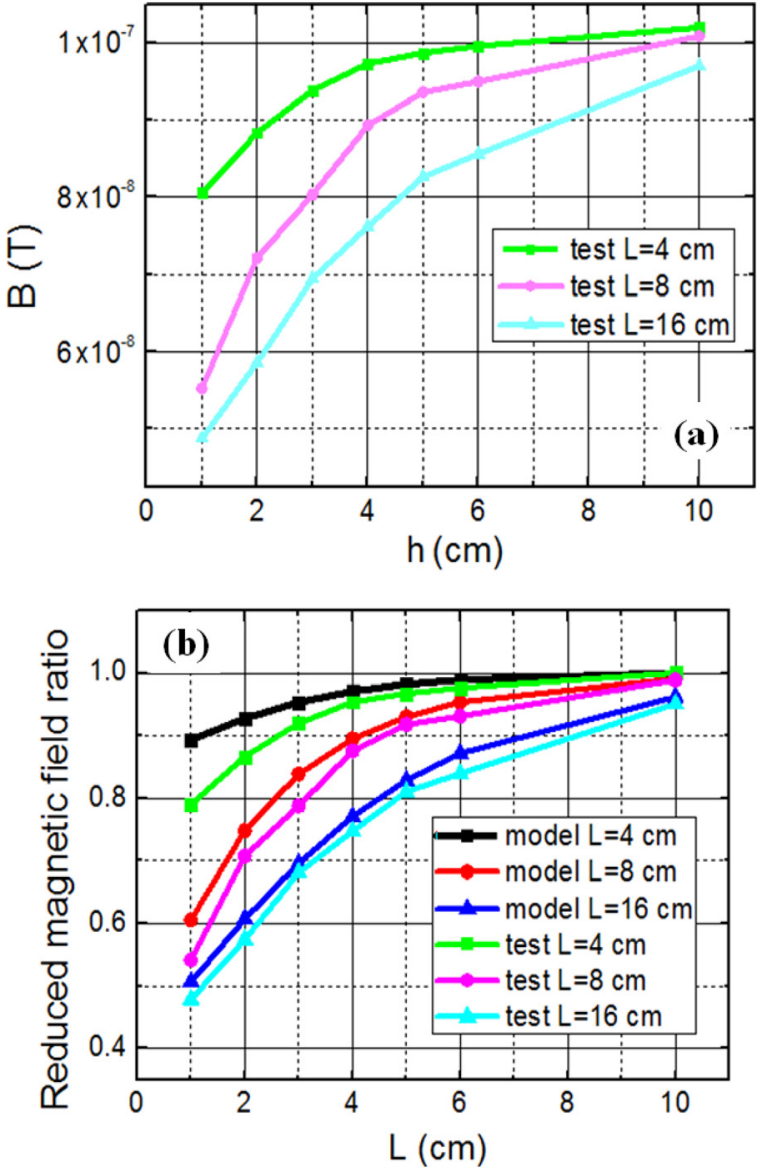

FIG. 3. (a) The magnetic field response of a ME sensor as a function of distance $\mathrm{h}$ for different lengths (L) for nearby metal plates under a constant incident AC magnetic field background produced by a solenoid coil. (b) Magnetic field decrement ratio caused by the magnetic flux distraction effect for experimental and simulated data.

ratio. Results from experiment and simulation can be seen to agree well with each other. The metal object diverts flux density from the ME sensor. This effect was found to decrease as the distance increased between the sensor and object, until the distance was sufficiently high that mutual inductance did not occur. It was also found that the flux distraction was more significant for larger masses. These variances in the observed $B$ field using highly sensitive ME sensors offer a new magnetometer technology that is promising for use in precise magnetic anomaly field detection to discriminate targets over a specific survey area that have different dimensions (masses) and scopes.

Two ME sensors S1 and S2 were then arranged with a baseline of $20 \mathrm{~cm}$. A sinusoid wave signal at $1 \mathrm{~Hz}$ was generated by a solenoid coil located $40 \mathrm{~cm}$ away to the central point of the two sensors. Next, a metal plate of size $8 \times 4 \times 0.3 \mathrm{~cm}^{3}$ was used to study the differential output (S1-S2) of the gradiometer as a function of distance. The metal was located close to $\mathrm{S} 1$ at a distance $=2 \mathrm{~cm}$. The distance was then gradually increased until a weak magnetic field disturbance could not be detected by the gradiometer. Figure 4(a) shows that the value of (S1-S2) was a maximum of $11 \mathrm{nT} / \sqrt{ } \mathrm{Hz}(f=1 \mathrm{~Hz})$ when the target was in close vicinity. The value of (S1-S2) then decreased rapidly to $60 \mathrm{pT} /{ }_{\sqrt{ }} \mathrm{Hz}$ as 

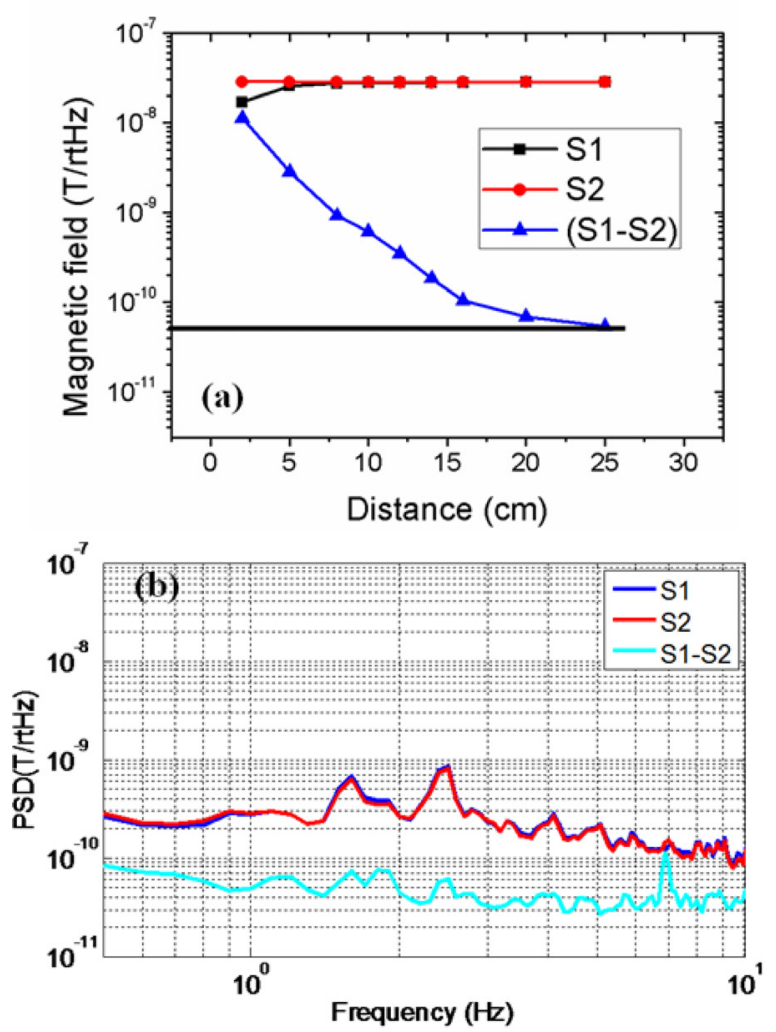

(c)
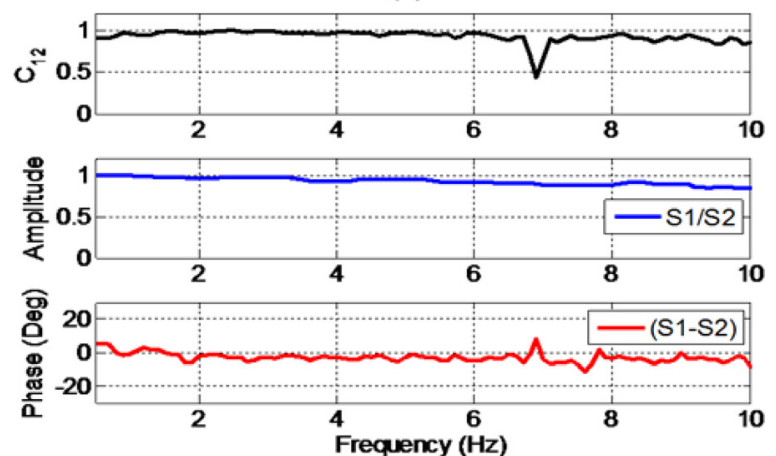

FIG. 4. (a) Gradiometer differential output (S1-S2) as a function of target distance for a $8 \times 4 \times 0.3 \mathrm{~mm}^{3}$ metal target. The amplitude (S1-S2) was a maximum of $11 \mathrm{nT} / \sqrt{ } \mathrm{Hz}$ at $f=1 \mathrm{~Hz}$ when the target was close. The strength of (S1-S2) then dropped rapidly to about $60 \mathrm{pT} /{ }_{\sqrt{ }} \mathrm{Hz}$ as the distance was increased to $20 \mathrm{~cm}$, above which point the differential output could not be detected from the noise. (b) Power spectra density (PSD) of individual ME sensors that have an equivalent background noise level of $0.2 \mathrm{nT} / \sqrt{ } \mathrm{Hz}$ for $1<f<10 \mathrm{~Hz}$. (c) Coherence between S1 and S2 outputs (top graph) in terms of their amplitude ratio (S1/S2) (middle graph) and phase discrepancy (S1-S2) (bottom graph).

the distance was increased to $20 \mathrm{~cm}$, where a differential output could not be discriminated from the background noise. The maximum distance that such a ME gradiometer can detect an object is directly dependent on the sensor's sensitivity and the background noise. Our findings show that a ME gradiometer can be exploited for efficient metal detection for distances of up to $20 \mathrm{~cm}$ (i.e., for a small $8 \times 4 \times 0.3 \mathrm{~cm}^{3}$ plate) with a limiting detection sensitivity of $60 \mathrm{pT} / \sqrt{ } \mathrm{Hz}$. This represents a remarkable two orders of magnitude enhancement of detection sensitivity over previous
Anisotropic magnetoresistance (AMR) gradiometers at low frequency. ${ }^{14}$

The gradiometer did not result in the complete rejection of environmental noise. To identify the factor limiting the gradiometer's detection sensitivity for practical use, we then performed a noise test in an open environment and performed a coherence analysis of the results. The gradiometer was placed with the same original baseline, but without a drive signal. Figure 4(b) shows a representative power spectral density (PSD) plot for both sensors of the gradiometer pair, which reveals an equivalent background noise level of $0.2 \mathrm{nT} / \sqrt{ } \mathrm{Hz}$ for $1<f<10 \mathrm{~Hz}$. The peaks at $1-3 \mathrm{~Hz}$ may result from a building vibration noise. The differential noise $(\mathrm{S} 1-\mathrm{S} 2)$ of the gradiometer was around $10 \times$ lower than that of the individual sensors, as the pair can reject environmental noises that are coherently shared between them. It should be noted that the differential noise (S1-S2) was only $50 \mathrm{pT} /{ }_{\sqrt{ } \mathrm{Hz}}$ at $f=1 \mathrm{~Hz}$, which infers a fair coherence between the two gradiometer outputs $\mathrm{S} 1$ and $\mathrm{S} 2$.

Finally, Figure 4(c) shows a coherence analysis between the $\mathrm{S} 1$ and $\mathrm{S} 2$ outputs in terms of their amplitude ratio $(\mathrm{S} 1 / \mathrm{S} 2)$ and phase discrepancy $(\mathrm{S} 1-\mathrm{S} 2)$. The coherence $C_{12}$ gives information about the fraction of the power spectrum of signal S1 that also appears in the power spectrum of signal $\mathrm{S} 2$. From the top graph in Fig. 4(c), one can clearly see that $C_{12}$ remains constant at one for $1<f<5 \mathrm{~Hz}$, indicating a good coherent relation between $\mathrm{S} 1$ and $\mathrm{S} 2$ which is also certified by an efficient noise rejection over this frequency range (see Figure 4). However, at higher frequencies of $5<f<10 \mathrm{~Hz}, C_{12}$ decreased some which can be attributed to a decreased amplitude ratio (middle graph of Fig. 4(c)) and phase difference (bottom graph of Fig. 4(c)). The amplitude ratio $(\mathrm{S} 1 / \mathrm{S} 2)$ refers to the relative strengths of the two outputs, which remained nearly unity for $f<5 \mathrm{~Hz}$ and decreased slightly to 0.9 for $f>5 \mathrm{~Hz}$. The phase difference represents the time lag between the two signals, which can be seen to be about $2^{\circ}-6^{\circ}$ over the entire frequency bandwidth in Figure 4(c). These results together indicate that the sensor pair did not reach their optimum performance. A small discrepancy in amplitude and phase resulted in reduced capability of the gradiometer pair to effectively reject common environment noise.

In summary, by utilizing the high permeability of Metglas, a magnetic flux distraction effect was found in ME laminate sensors in response to the presence of metallic objects. This flux distraction effect was dependent on distance between sensor and object, and the volume of the object. The principle can be employed for stable ferromagnetic dipole detection using Metglas-based ME sensors, and is free of any unwanted motion over a survey area that could introduce vibration noises sources that degrade sensor performance. It has been found both theoretically and experimentally that ME sensors have precise magnetic field variances with respect to minute flux distraction effects resulting from changing target dimensions and distances. After rejecting common environmental noise, ME gradiometers can achieve a high detection sensitivity of $60 \mathrm{pT} / \sqrt{ } \mathrm{Hz}$ at quasistatic frequencies, which offers a viable solution for magnetic anomaly detection in a stationary operating mode. 
The authors thank the Office of Naval Research for funding.

${ }^{1}$ S. Cheong and M. Mostovoy, Nature Mater. 6, 13 (2007).

${ }^{2}$ C. Ederer and N. A. Spaldin, Nature Mater. 3, 849 (2004).

${ }^{3}$ J. Gao, Z. Wang, Y. Shen, M. Li, Y. Wang, P. Finkel, J. Li, and D. Viehland, Mater. Lett. 82, 178 (2012); Y. Shen, K. L. McLaughlin, J. Gao, D. Gray, L. Shen, Y. Wang, M. Li, D. Berry, J. Li, and D. Viehland, Smart Mater. Struct. 21(6), 065007 (2012); Y. Shen, K. McLaughlin, J. Gao, D. Gray, D. Hasanyan, Y. Wang, J. Li, and D. Viehland, IEEE Trans. Ultrason., Ferroelectr. Freq. Control 59(9), 2111 (2012).

${ }^{4}$ Y. Shen, J. Gao, L. Shen, D. Gray, J. Li, P. Finkel, D. Viehland, X. Zhuang, S. Saez, and C. Dolabdjian, Sens. Actuators, A 171(2), 63 (2011).

${ }^{5}$ Z. Fang, S. G. Lu, F. Li, S. Datta, Q. M. Zhang, and M. El Tahchi, Appl. Phys. Lett. 95(11), 112903 (2009).
${ }^{6}$ J. Gao, D. Gray, Y. Shen, J. Li, and D. Viehland, Appl. Phys. Lett. 99(15), 153502 (2011).

${ }^{7}$ Y. Shen, J. Gao, D. Hasanyan, Y. Wang, M. Li, J. Li, and D. Viehland, Smart Mater. Struct. 21(11), 115007 (2012).

${ }^{8}$ D. S. Parasnis, Principles of Applied Geophysics, 5th ed. (Chapman \& Hall, 1997).

${ }^{9}$ S. Dong, J. Zhai, J. Li, and D. Viehland, Appl. Phys. Lett. 89(25), 252904 (2006); J. Zhai, Z. Xing, S. Dong, J. Li, and D. Viehland, J. Am. Ceram. Soc. 91(2), $351(2008)$.

${ }^{10}$ Y. Wang, D. Gray, D. Berry, J. Gao, M. Li, J. Li, and D. Viehland, Adv. Mater. 23(35), 4111 (2011).

${ }^{11}$ J. Das, J. Gao, Z. Xing, J. F. Li, and D. Viehland, Appl. Phys. Lett. 95(9), 092501 (2009).

${ }^{12}$ Z. Xing, J. Li, and D. Viehland, Appl. Phys. Lett. 91(18), 182902 (2007).

${ }^{13}$ Y. Shen, J. Gao, Y. Wang, J. Li, and D. Viehland, Appl. Phys. Lett. 100(17), 173505 (2012).

${ }^{14}$ J. Vyhnánek, M. Janošek, and P. Ripka, Sens. Actuators, A 186, 100 (2012). 